\title{
Effects of Surface Functional Groups on Electron Transfer at Liquid-Solid Interfacial Contact Electrification
}

Shiquan $\operatorname{Lin}^{\dagger, \ddagger, \triangle}$, Mingli Zheng ${ }^{\dagger, \ddagger, \triangle}$, Jianjun Luo ${ }^{\dagger, \ddagger}$, , Zhong Lin Wang, ${ }^{*}{ }^{\dagger, \S}$

$\uparrow$ Beijing Institute of Nanoenergy and Nanosystems, Chinese Academy of Sciences, Beijing 100083, P. R. China

† School of Nanoscience and Technology, University of Chinese Academy of Sciences, Beijing 100049, P. R. China

$\S$ School of Materials Science and Engineering, Georgia Institute of Technology, Atlanta, GA 30332-0245, USA

$\triangle$ These authors contributed equally to this work. 

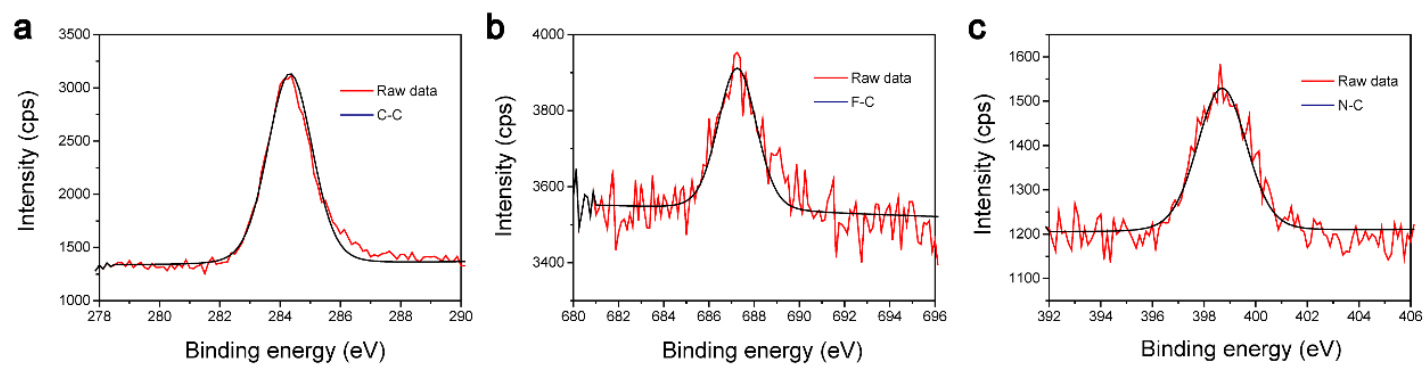

Figure S1. The XPS characterization of the $\mathrm{SiO}_{2}$ surfaces. The XPS spectrums of the (a) unmodified $\mathrm{SiO}_{2}$ surface, $\mathrm{SiO}_{2}$ surfaces modified with (b) perfluoro-dodecyl, (c) aminopropyl.

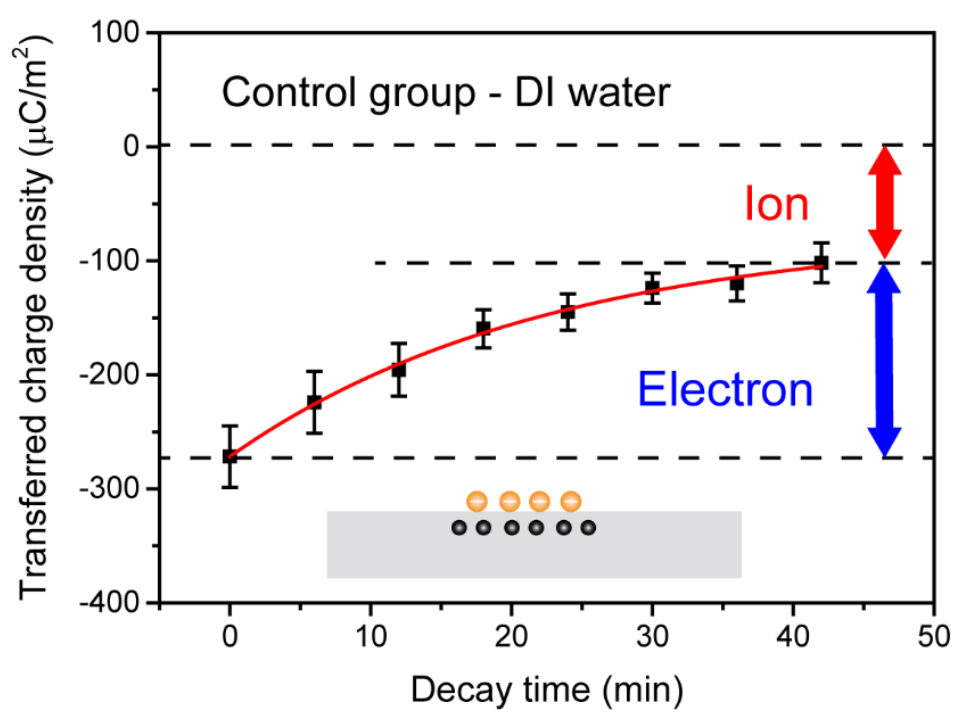

Figure S2. Temperature effect on the $\mathrm{CE}$ between the DI water and unmodified $\mathrm{SiO}_{2}$ (control group). The decay of the $\mathrm{CE}$ charges (induced by contacting with the DI water at room temperature) on the unmodified $\mathrm{SiO}_{2}$ surfaces at $413 \mathrm{~K}$.
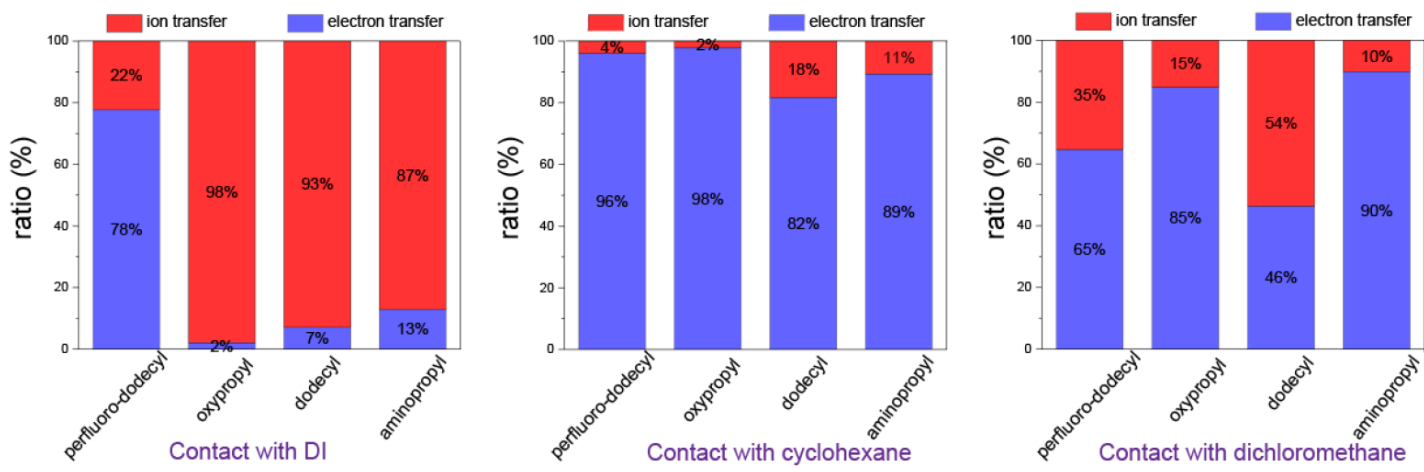

Figure S3. The ratio of ion transfer to electron transfer in the contact electrification between liquid and modified $\mathrm{SiO}_{2}$. 

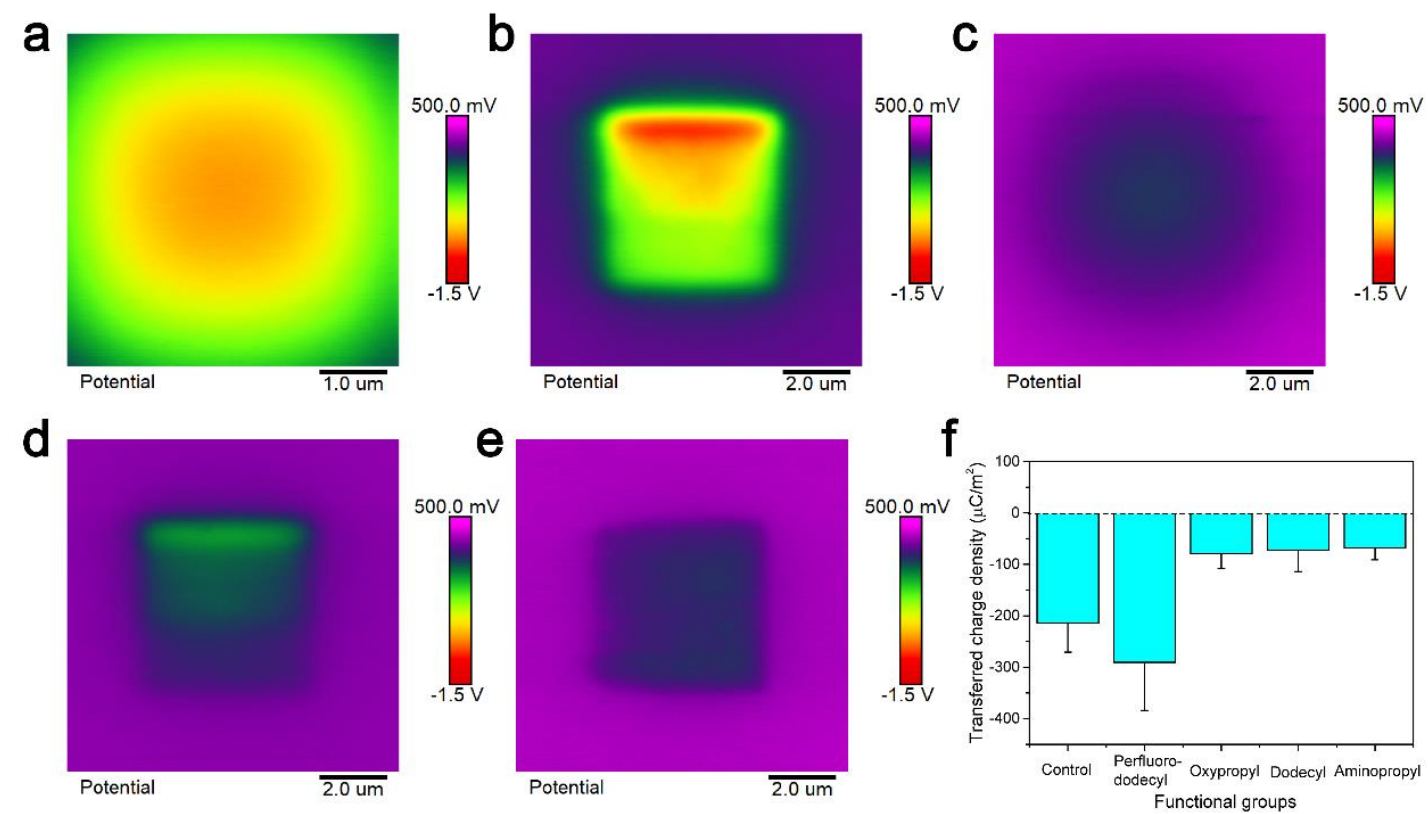

Figure S4. The CE between modified $\mathrm{SiO}_{2}$ and $\mathrm{Au}$ coated tip. The surface potential of the (a) unmodified $\mathrm{SiO}_{2}$, (b) perfluoro-dodecyl, (c) oxypropyl, (d) dodecyl and (e) aminopropyl modified $\mathrm{SiO}_{2}$ surfaces after contact by Au coated tip. (f) Effects of functional groups on the transferred charge density between $\mathrm{SiO}_{2}$ and Au coated tip.

a

Control group

Contact angle $=55.58$

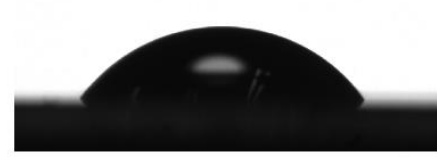

d

Dodecyl

Contact angle $=98.83$

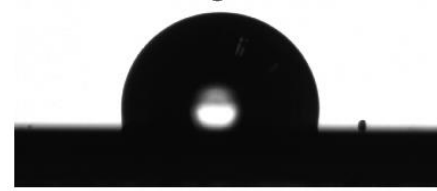

b Perfluorododecyl

Contact angle $=92.25$

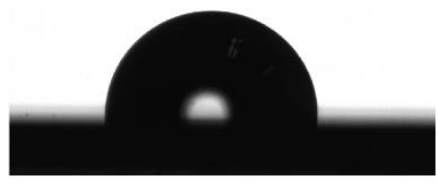

e Aminopropyl

Contact angle $=80.79$

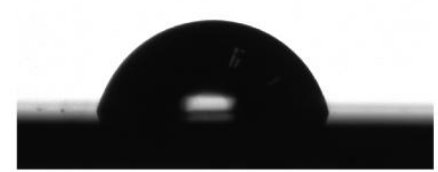

\section{Oxypropyl}

Contact angle $=58.44$
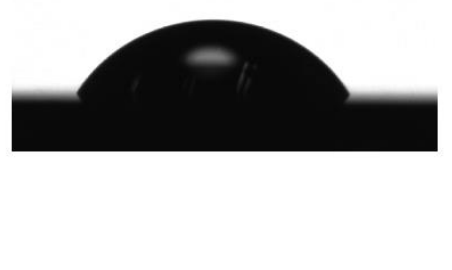

Figure S5. The water contact angle of the (a) unmodified $\mathrm{SiO}_{2}$, (b) perfluoro-dodecyl, (c) oxypropyl, (d) dodecyl and (e) aminopropyl modified $\mathrm{SiO}_{2}$ surfaces. 